\title{
MULTIPLICATIONS ON THE LINE
}

J. G. HORNE, JR.

Mostert and Shields characterized ordinary multiplication on $[0, \infty)$ and briefly considered multiplication on $E_{1}=(-\infty, \infty)$ [2]. However, a characterization of ordinary multiplication on $E_{1}$ has apparently not been given. In this note, such a characterization is presented. In addition, all other topological semigroups on $E_{1}$ are determined in which 0 and 1 play their usual roles and whose multiplications agree on $[0, \infty)$ with ordinary multiplication. It is an interesting corollary of these results that Faucett's characterization of ordinary multiplication on the closed unit interval carries over to $E_{1}[1]$.

The author is happy to acknowledge helpful correspondence he has had concerning this note with $\mathrm{J}$. W. Ellis and P. S. Mostert.

In general, $\left(E_{1}, 0\right)$ denotes a topological semigroup on $E_{1}$ in which $O$ is a continuous associative multiplication. The ordinary product of $x$ and $y$ is written $x y$, and $\left(E_{1}, \cdot\right)$ denotes the semigroup on $E_{1}$ under ordinary multiplication. We always assume that if $x, y \in[0, \infty)$, then $x \circ y=x y$. In addition, we suppose that 0 and 1 act as zero and identity respectively for $\left(E_{1}, 0\right)$. Recall, from [2], that an iseomorphism is a semigroup isomorphism which is also a homeomorphism.

We shall want to refer to the following classes of topological semigroups on $E_{1}$. Except for $\left(E_{1}, \cdot\right)$, these turn out to be the only possible topological semigroups on $E_{1}$ subject to the above restrictions.

Example 1. For fixed $\alpha>0$, define multiplication $x \nu y$ in $E_{1}$ as follows:

(i) for $x \in E_{1}, y \in[0, \infty), x \nu y=x y$,

(ii) for $x \in[0, \infty), y \in(-\infty, 0), x \nu y=x^{\alpha} y$,

(iii) for $x, y \in(-\infty, 0), x \nu y=0$.

Example 2. Define multiplication $x \tau y$ in $E_{1}$ as follows:

(i) for $x \in[0, \infty), y \in E_{1}, x \tau y=y \tau x=x y$,

(ii) for $x, y \in(-\infty, 0), x \tau y=-(x y)$.

Hereafter we shall abbreviate $(0, \infty)$ to $P$ and let $P^{-}=[0, \infty)$, $N=(-\infty, 0)$ and $N^{-}=(-\infty, 0]$.

It should be remarked that no two of the semigroups of Example 1 are iseomorphic. For suppose $\alpha$ and $\beta$ are two positive numbers and that $\nu_{1}$ and $\nu_{2}$ are the corresponding multiplications. Suppose $T:\left(E_{1}, \nu_{1}\right) \rightarrow\left(E_{1}, \nu_{2}\right)$ is an iseomorphism. Now the elements in $P^{-}$can

Received by the editors March 28, 1958. 
be recognized in any of the semigroups in Example 1 since these are the only elements with square roots. Hence $T$ is an iseomorphism on $P$ - to itself. Therefore there is a number $\gamma>0$ such that $T(t)=t^{\gamma}$ if $t \geqq 0$. Now for any such $t$, we have

$$
\begin{aligned}
T\left(t \nu_{1}(-1)\right) & =T\left((-1) \nu_{1} t^{\alpha}\right)=T(-1) \nu_{2} T\left(t^{\alpha}\right)=T(-1) \nu_{2}\left(t^{\alpha}\right)^{\gamma} \\
& =T(-1)\left(t^{\alpha \gamma}\right) .
\end{aligned}
$$

The last equality is justified since $T(-1) \in(-\infty, 0)$. On the other hand,

$$
T(t) \nu_{2} T(-1)=\left(t^{\gamma}\right) \nu_{2} T(-1)=T(-1)\left(t^{\gamma}\right)^{\beta} .
$$

Therefore $t^{\alpha \gamma}=t^{\gamma \beta}$ and hence $\alpha=\beta$.

We now proceed to derive the main theorems. We first prove several lemmas which hold without further restrictions on $\left(E_{1}, 0\right)$.

Lemma 1. For any pair of distinct elements $a, b \in N$, there are numbers $t, t^{\prime} \in P$, either both in $(0,1)$ or both in $(1, \infty)$, such that $a=t \circ b=b \circ t^{\prime}$.

Proof. For any $x \in N$, right and left multiplications by $x$ are continuous functions which map 0 into 0 and 1 into $x$. Therefore every point in the interval $(x, 0)$ is the image of a number in $(0,1)$. Now either $a \in(b, 0)$ or $b \in(a, 0)$. In the first case, take $x=b$ and obtain $t, t^{\prime} \in(0,1)$ such that $a=t \circ b=b \circ t^{\prime}$. In the second case, take $x=a$ and obtain $t, t^{\prime} \in(0,1)$ such that $a=\left(t^{-1}\right) \circ b=b \circ\left(t^{\prime-1}\right)$.

Lemma 2. If $x \in N$ and $x^{-1}$ exists then $x^{-1} \in N$.

Proof. If $x^{-1} \in P$ then $x=\left(x^{-1}\right)^{-1} \in P$.

Lemma 3. If $x \in N$ and $t \in P$ then $x \circ t$ and $t \circ x \in N$.

Proof. If $x \circ t \in P^{-}$and $t \in P$ then $x=(x \circ t) \circ t^{-1} \in P^{-}$. A similar statement holds if $t \circ x \in P^{-}$.

Lema 4. If $x \in N$ and $t, t^{\prime}$ are distinct elements of $P$ then $t \circ x$ $\neq t^{\prime} \circ x$ and $x \circ t \neq x \circ t^{\prime}$.

Proof. We prove only that $t \circ x \neq t^{\prime} \circ x$, and for this it is sufficient to prove that $t \neq 1$ implies $t \circ x \neq x$. Suppose, on the contrary, that $t \circ x=x$. Then $t^{-1} \circ x=x$ also. Hence we may as well assume $t<1$. Now for each positive integer $n, t^{n} \circ x=x$. But $t^{n} \circ x \rightarrow 0$, which is a contradiction since $x \in N$.

Lemma 5. If all members of $P$ commute with some element $v \in N$, then $\left(E_{1}, 0\right)$ is a commutative semigroup.

Proof. Suppose $t \circ v=v \circ t$ for all $t \in P$. For any $x, y \in N$ there are numbers $t_{1}, t_{2} \in P$ such that $x=t_{1} \circ v$ and $y=t_{2} \circ v$. If $t \in P$ then 
$t \circ x=t \circ\left(t_{1} \circ v\right)=\left(t t_{1}\right) \circ v=v \circ\left(t t_{1}\right)=v \circ\left(t_{1} t\right)=\left(v \circ t_{1}\right) \circ t=\left(t_{1} \circ v\right) \circ t$ $=x \circ t$, so members of $P$ commute with all members of $N$. On the other hand: $x \circ y=\left(t_{1} \circ v\right) \circ\left(t_{2} \circ v\right)=\left(t_{1} \circ v\right) \circ\left(v \circ t_{2}\right)=t_{2} \circ\left(t_{1} \circ v\right) \circ v$ $=t_{2} \circ\left(v \circ t_{1}\right) \circ v=\left(t_{2} \circ v\right) \circ\left(t_{1} \circ v\right)=y \circ x$, so members of $N$ commute with each other.

Lemma 6. There is a positive number $\alpha$ such that $t \circ x=x \circ t^{\alpha}$ for all $x \in N^{-}, t \in P^{-}$.

Proof. Fix $a \in N$ and set $f(t)=a \circ t$ and $g(t)=t \circ a$ for $t \in P^{-} . f$ and $g$ are continuous functions from $P^{-}$to $N^{-}$. By Lemma 1, they map onto $N^{-}$and by Lemma 4 , they are one-to-one. Thus $f^{-1} g$ is a continuous function from $P^{-}$to itself which is actually multiplicative. For let $t, t^{\prime} \in P^{-}$and let $s=f^{-1} g(t)$ and $s^{\prime}=f^{-1} g\left(s^{\prime}\right)$; that is, $t \circ a=a \circ s$ and $t^{\prime} \circ a=a \circ s^{\prime}$. Now $f^{-1} g\left(t t^{\prime}\right)=f^{-1}\left(\left(t t^{\prime}\right) \circ a\right)=f^{-1}\left(t \circ\left(t^{\prime} \circ a\right)\right)$ $=f^{-1}\left(t \circ\left(a \circ s^{\prime}\right)\right)=f^{-1}\left((t \circ a) \circ s^{\prime}\right)=f^{-1}\left((a \circ s) \circ s^{\prime}\right)=f^{-1}\left(a \circ\left(s s^{\prime}\right)\right)=s s^{\prime}$ $=f^{-1} g(t) f^{-1} g\left(t^{\prime}\right)$. Therefore there is a number $\alpha$ such that for all $t \in P, f^{-1} g(t)=t^{\alpha}$. Since $f^{-1} g$ is continuous at zero, $\alpha$ is positive. By the definitions of $f$ and $g$ we have $t \circ a=a \circ t^{\alpha}$ for all $t \in P^{-}$.

Now let $x \in N^{-}$be arbitrary. There is $t_{1} \in P^{-}$such that $x=t_{1} \circ a$. Therefore $x=a \circ t_{1}^{\alpha}$ and for $t \in P^{-}, t \circ x=t \circ\left(t_{1} \circ a\right)=\left(t t_{1}\right) \circ a=a \circ\left(t t_{1}\right)^{\alpha}$ $=a \circ\left(t^{\alpha} \circ t_{1}^{\alpha}\right)=\left(a \circ t_{1}^{\alpha}\right) \circ t^{\alpha}=x \circ t^{\alpha}$. This completes the proof of the lemma.

THEOREM 1. If there is at least one pair of elements $x_{1}, x_{2} \in N$ such that $x_{1} \circ x_{2} \in P$, then $\left(E_{1}, \circ\right)$ is iseomorphic to $E_{1}$ under ordinary multiplication.

Proof. Let $x, y$ be an arbitrary pair of elements in $N$. Write $x=t_{1} \circ x_{1}$ and $y=x_{2} \circ t_{2}$ with $t_{1}, t_{2} \in P$. Then $x \circ y=\left(t_{1} \circ x_{1}\right) \circ\left(x_{2} \circ t_{2}\right)$ $=t_{1} \circ\left(x_{1} \circ x_{2}\right) \circ t_{2} \in P$. Thus, the product of any pair of elements in $N$ belongs to $P$. In particular, $x \circ x \in P$, so there is $t \in P$ such that $(x \circ x) \circ t=t \circ(x \circ x)=1$. Therefore, $x \circ(x \circ t)=(t \circ x) \circ x=1$, so every element of $N$ has an inverse which belongs to $N$, by Lemma 2 . Now consider the function $f(x)=x \circ x . f$ is a continuous function from $N$ into $P$ having the property that if $t$ belongs to range $f$ then so does $t^{-1}$. Hence 1 belongs to range $f$; that is, there is at least one $u \in N$ such that $u \circ u=1$. If $x$ is any other element of $N$ then there are elements $t, t^{\prime} \in P$, either both in $(0,1)$ or both in $(1, \infty)$, such that $x=t \circ u=u \circ t^{\prime}$. Hence $x \circ x=(t \circ u) \circ\left(u \circ t^{\prime}\right)=t \circ(u \circ u) \circ t^{\prime}=t t^{\prime}$ $\neq 1$. In other words, $u$ is the only square root of 1 which belongs to $N$. It follows that $u$ commutes with all elements of $P$. For if $t \in P$ then $t \circ u \circ t^{-1} \in N$, and $\left(t \circ u \circ t^{-1}\right) \circ\left(t \circ u \circ t^{-1}\right)=1$. Hence $t \circ u \circ t^{-1}=u$, or $t \circ u=u \circ t$. From this we infer that $\left(E_{1}, 0\right)$ is a commutative semigroup by Lemma 5 . 
Now set $I(x)=x$ if $x \in P^{-}$, and $I(x)=-(u \circ x)$ if $x \in N$.

It is straightforward to show that $I:\left(E_{1}, 0\right) \rightarrow\left(E_{1}, \cdot\right)$ is an isomorphism and a homeomorphism, and the details are omitted.

Next we consider the possibilities in case the hypothesis of Theorem 1 is not satisfied-that is, in case $N^{-}$is a sub-semigroup of $\left(E_{1}, 0\right)$.

THEOREM 2. If $u \circ v=0$ for some $u, v \in N$ then $x \circ y=0$ for all $x, y \in N$ and $\left(E_{1}, 0\right)$ is iseomorphic to one of the semigroups $\left(E_{1}, \nu\right)$ of Example 1.

Proof. Suppose $u \circ v=0$ and write $v=u \circ t$ with $t \in P$. Then $0=u \circ v=u \circ(u \circ t)=(u \circ u) \circ t$, so $u \circ u=0$. Now for any $x, y \in N$, we have $x=s \circ u$ and $y=u \circ s^{\prime}$ for some $s, s^{\prime} \in P$. Thus $x \circ y=(s \circ u)$ $\circ\left(u \circ s^{\prime}\right)=s \circ(u \circ u) \circ s^{\prime}=0$.

By Lemma 6, there is $\alpha>0$ such that $t \circ x=x \circ t^{\alpha}$ for all $x \in N^{-}$, $t \in P^{-}$. Once again take advantage of the fact that if $a$ is a fixed element of $N$ then every element $x$ of $N$ can be written $x=t \circ a=a \circ t^{\alpha}$ for some (unique) $t \in P$. Now define $T: E_{1} \rightarrow E_{1}$ by

$$
T(x)=-t^{\alpha} \text { if } x \in N \text {, and } T(x)=x \text { if } x \in P^{-} .
$$

Then $T$ is an iseomorphism from $\left(E_{1}, 0\right)$ onto the semigroup $\left(E_{1}, \nu\right)$ of Example 1 which corresponds to the present value of $\alpha$. Certainly $T$ is a homeomorphism onto $E_{1}$. Let $x, y \in E_{1}$. If both $x, y$ belong to $N$ or if both belong to $P^{-}$, then obviously $T(x \circ y)=T(x) \nu T(y)$. Suppose $x \in N$ and $y \in P^{-}$with $x=t \circ a$. Then $T(y \circ x)=T(y \circ(t \circ a))$ $=T((y t) \circ a)=-(y t)^{\alpha}$, while $T(y) \nu T(x)=y \nu\left(-t^{\alpha}\right)=-\left(y^{\alpha} t^{\alpha}\right)$. On the other hand, $T(x \circ y)=T((t \circ a) \circ y)=T(t \circ(a \circ y))=T\left(t \circ\left(y^{\beta} \circ a\right)\right)$ where $\beta \alpha=1$. Thus $T(x \circ y)=-\left(t y^{\beta}\right)^{\alpha}=-t^{\alpha} y$. Finally, $T(x) \nu T(y)$ $=\left(-t^{\alpha}\right) \nu y=-t^{\alpha} y$ and the proof of the theorem is complete.

Theorem 3. If $x, y \in N$ imply $x \circ y \in N$, then $\left(E_{1}, 0\right)$ is iseomorphic to the semigroup of Example 2.

Proof. For any $a, b \in N$, we may write $b=(a \circ a) \circ t$ and $b$ $=t^{\prime} \circ(a \circ a)$ for some $t, t^{\prime} \in P$. Hence $b=a \circ(a \circ t)$ and $b=\left(t^{\prime} \circ a\right) \circ a$, and $a \circ t$ and $t^{\prime} \circ a$ belong to $N$. Thus the equations $a \circ x=b$ and $y \circ a=b$ always have solutions in $N$. Therefore $(N, 0)$ is a group and hence possesses exactly one idempotent $e$ which is an identity. Since 0 is a zero for $N^{-},\left(N^{-}, 0\right)$ is a topological semigroup with zero and identity and no other idempotents. It follows from [1, Theorem A] that $\left(N^{-}, 0\right)$ is iseomorphic to $[0, \infty)$ under ordinary multiplication. In particular, multiplication is commutative on $N$. Now for any $t \in P,(t \circ e) \circ t^{-1}=(t \circ e \circ e) \circ t^{-1}=(t \circ e) \circ\left(e \circ t^{-1}\right)=\left(e \circ t^{-1}\right) \circ(t \circ e)$ $=e$, so $t \circ e=e \circ t$. Hence $\left(E_{1}, \circ\right)$ is a commutative semigroup. Now define $T$ as follows: If $x \in N$ then $x=t \circ e$ for some $t \in P$. Set 


$$
T(x)=-t \text { if } x \in N \text { and } T(x)=x \text { if } x \in P^{-} .
$$

It is easy to check that $T$ is an iseomorphism between $\left(E_{1}, 0\right)$ and the semigroup $\left(E_{1}, \tau\right)$ of Example 2. The proof of the theorem is complete.

Finally, note that the semigroup of Example 2 has the idempotent -1 , while all of the semigroups of Example 1 contain nilpotent elements. Hence, we have the following extension to $E_{1}$ of Faucett's characterization of ordinary multiplication on the closed unit interval [1].

Corollary. If $S$ is a topological semigroup on $E_{1}$ which possesses a zero and identity and no other idempotents, and if $S$ contains no nonzero nilpotent elements, then $S$ is iseomorphic to $E_{1}$ under ordinary multiplication.

\section{REFERENCES}

1. W. M. Faucett, Compact semigroups irreducibly connected between two idempotents, Proc. Amer. Math. Soc. vol. 6 (1955) pp. 741-747.

2. P. S. Mostert and A. L. Shields, On a class of semigroups on $E_{n}$, Proc. Amer. Math. Soc. vol. 7 (1956) pp. 729-734.

UNIVERSITY OF KENTUCKY 